\title{
Strates
}

STRATES Matériaux pour la recherche en sciences sociales

$7 \mid 1993$

Témoins du monde : Bulgarie, identités chinoises, explorer l'île de France

\section{La Bulgarie aujourd'hui : un cas dans les Balkans?}

\section{Marin Batchvarov}

\section{(2) OpenEdition \\ Journals}

Édition électronique

URL : http://journals.openedition.org/strates/1112

ISSN : 1777-5442

\section{Éditeur}

Laboratoire Ladyss

Édition imprimée

Date de publication : 30 juin 1993

ISSN : 0768-8067

\section{Référence électronique}

Marin Batchvarov, «La Bulgarie aujourd'hui : un cas dans les Balkans? », Strates [En ligne], 7 | 1993, mis en ligne le 20 décembre 2005, consulté le 07 septembre 2020. URL : http:// journals.openedition.org/strates/1112

Ce document a été généré automatiquement le 7 septembre 2020

Tous droits réservés 


\title{
La Bulgarie aujourd'hui : un cas dans les Balkans?
}

\author{
Marin Batchvarov
}

\section{NOTE DE L'ÉDITEUR}

Ce texte a été revu par Pierre Lenormand. Dans le cadre du séminaire « Europe » du laboratoire STRATES, Marin Batchvarov a donné à l'Institut de Géographie le 13 avril 1992 une conférence sur le thème : «La Bulgarie au carrefour des meilleures Europes possibles ». C'est le texte de cette conférence qu'il a bien voulu reprendre lui-même, puis actualiser, que nous proposons : il apporte sur ce pays méconnu des informations et une opinion qui devraient nourrir le dialogue poursuivi depuis de nombreuses années, autour et au sein du laboratoire, entre les deux côtés de l'Europe.

La Bulgarie dans le nouveau contexte géopolitique

1 Quelles que soient les causes de l'échec de l'expérience et du modèle soviétique - motifs économiques, idéologiques et politiques internes, ou efficacité de la stratégie du containment $^{1}$ pratiqué après la deuxième guerre mondiale par les Américains et leurs alliés - l'empire qui symbolise le fameux Heartland du géographe britannique $\mathrm{H}$. Mackinder ${ }^{2}$ n'existe plus. La "périphérie insulaire $d u$ monde» l'a emporté sur la «Mitteleuropa » d'abord, sur l'Empire soviétique ensuite.

2 Mackinder justement pensait, non sans raison, que le bassin de la mer Noire et du Danube devait plutôt être rattaché au Heartland qu'à la périphérie anglo-saxonne. Maintenant, avec l'éclatement de l'URSS, cette région intermédiaire est profondément troublée, d'autant que d'énormes problèmes nationaux, politiques et culturels s'y posent. Balkans et Caucase, avec leurs conflits internes hérités des siècles passés, ont été marginalisés et contrôlés par les superpuissances à la faveur de la guerre froide. Mais avec la décadence des blocs militaires, les vieux fantômes des rivalités se sont brusquement réveillés ${ }^{3}$. 
3 Dans ce contexte géopolitique, la Bulgarie est un cas particulier: les profonds changements socio-économiques et politiques survenus dès 1989 se sont déroulés sans représailles sanglantes, et malgré d'affreux problèmes économiques le pays est resté relativement stable. Pourtant, il serait naïf de croire qu'il n'y a pas de forces qui peuvent tenter une déstabilisation: on a pu observer l'activation des thèmes nationalistes bulgares et turcs, et certains politiciens, jouant sur le thème du populisme, en profitent. Malgré tout je suis convaincu que le danger de guerre civile est passé et que la construction d'une société plus démocratique, mais aussi plus stratifiée socialement, a commencé. Le pays sort de son isolement et cherche à se repositionner dans le monde. Mais sur quelle piste géopolitique?

Une aspiration forte à l'intégration européenne

4 Je ne pense pas qu'un modèle américain ou sud-coréen puisse être sérieusement envisagé pour la Bulgarie. Il existe dans les Balkans de profondes matrices historiques et socioculturelles qui les relient à l'Europe occidentale et méditerranéenne, à la puissance orthodoxe (la Russie) et au Proche-Orient.

Figure 1 : BULGARIE : QUELLES PISTES GÉOPOLITIQUES?

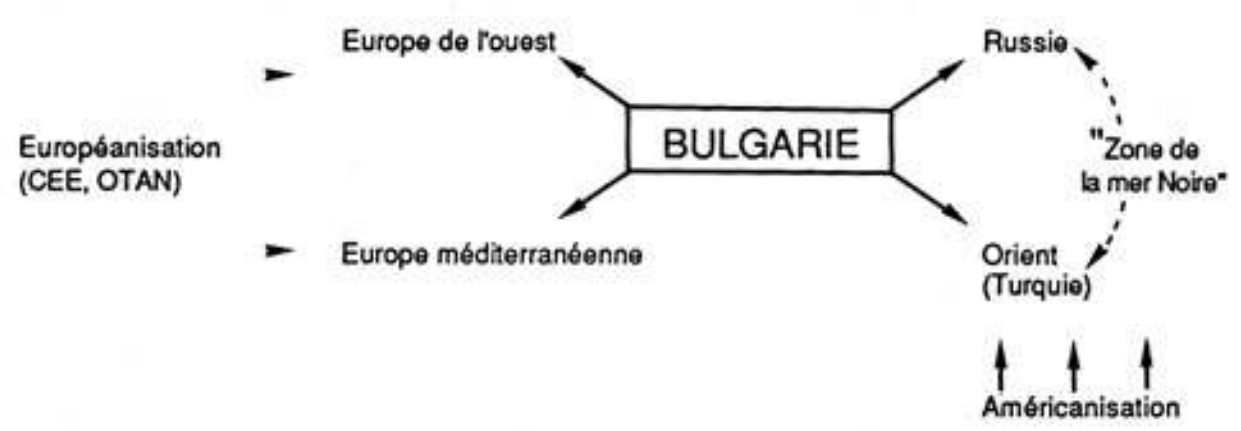

5 La péninsule a comme on le sait appartenu à l'Empire ottoman pour une longue période, durant laquelle l'Europe s'est considérablement développée tandis que les Balkans sombraient dans un profond marasme. On ne peut donc envisager d'autres pôles géopolitiques d'intégration - ou d'alignement - que les trois cités plus haut, qu'il est possible de ramener, compte tenu de l'état actuel de la Russie, à deux options :

- une option orientale, personnifiée par une Turquie en progrès et la fameuse « zone de la mer Noire » : celle-ci incluant la Roumanie, la Russie et d'autres républiques postsoviétiques limitrophes, on aurait là une combinaison des pôles d'attraction russe et oriental, dont la Turquie serait, dans cette période historique, le moteur. N'oublions pas que ce projet reçoit un fort soutien américain, lié à l'importance stratégique exceptionnelle de la Turquie dans le dispositif de l'OTAN. La piste « pontique » est pour la Bulgarie attractive sur le plan économique et commercial, mais répulsive sur les plans politique, ethnique et culturel.

7 - la volonté claire et affirmée de la population bulgare est celle de l'option occidentale et européenne, qui se traduit dans la volonté du gouvernement d'aboutir à une association avec la Communauté Économique Européenne et avec l'OTAN. Il faut ici souligner le climat psychologique dans un pays longtemps protégé par le Pacte de Varsovie : face à une Turquie puissante et avec une forte minorité musulmane dans leur pays, la plupart des Bulgares voient dans l'OTAN et l'Europe une protection, une 
garantie géopolitique. La signature en décembre 1992 de l'accord d'association avec la CEE, après plusieurs mois de négociations, est un premier pas dans la voie de l'intégration européenne.

8 Dans ce contexte, la compréhension entre les pays balkaniques est un élément important pour l'intégration avec l'Europe, et la Bulgarie s'efforce d'y contribuer. S'il existe beaucoup d'intérêts communs entre les pays de la péninsule, il est vrai qu'il existe aussi des sujets de conflit, et tout particulièrement le problème macédonien ${ }^{4}$. La Bulgarie a reconnu l'État macédonien souverain, ce qui a automatiquement détérioré ses relations avec la Serbie, mais aussi avec la Grèce avec laquelle la coopération s'est développée depuis une dizaine d'années. Nous comprenons la position grecque, d'ailleurs nous redoutons également les revendications futures de Skopje sur la Macédoine bulgare, mais ne pas reconnaître la volonté qui s'est très majoritairement exprimée dans cette république n'aurait pu qu'aggraver la situation géopolitique de la région. Il faut ajouter enfin que si les Macédoniens ont bien les mêmes racines que les Bulgares, il n'existe même pas une voie ferrée entre les deux pays et les échanges s'en trouvent très limités : économiquement, c'est par les vallées du Vardar et de la Morava que la Macédoine de Skopje se rattache à l'Europe et au monde. Le problème de l'existence d'une minorité macédonienne ne se pose pas en Bulgarie : les habitants de la partie bulgare de la province historique de Macédoine se sentent Bulgares 5 .

9 Signalons toutefois que, conséquence d'une histoire tourmentée, $20 \%$ de la population bulgare descend des réfugiés originaires de la Macédoine grecque et yougoslave, de la Thrace turque ou grecque, et de la Dobroudja roumaine.

Une crise profonde et inédite...

10 Après quarante-cinq ans de développement comme pays socialiste du bloc soviétique, la Bulgarie se trouve dans une situation extrêmement difficile. La crise économique qui affecte des secteurs essentiels de l'économie, et dont les premiers symptômes étaient visibles dès la seconde moitié des années soixante-dix est générale, profonde, structurelle. Malgré ses multiples mais « cosmétiques » efforts de réforme, le pouvoir communiste n'était pas capable d'introduire les changements nécessaires, qui touchaient la logique du système et le monopole politique du parti.

11 L'effondrement des marchés extérieurs traditionnels (URSS et pays du CAEM), la dévaluation du lev et la réduction du pouvoir d'achat qui s'en est suivie ont accéléré la chute de la production amorcée dès 1989: en 1992, la production industrielle ne représente plus que $60 \%$ de ce qu'elle était en 1989, l'industrie lourde (énergie, sidérurgie, raffinage, mines et armement) étant particulièrement frappée. Le chômage atteint $20 \%$ de la population en âge d'activité, et l'inflation, même ralentie, a creusé l'écart entre les salaires et les prix.

12 Dans le même temps il y a des secteurs et des entreprises dont la capacité n'est pas utilisée rationnellement. C'est bien une crise de sous-production et non pas, comme cela est typique des pays développés, de surproduction. La structure des activités économiques du pays était peu conforme à sa taille et à ses ressources. La création d'un grand secteur d'industrie lourde fut de ce point de vue une erreur fondamentale. En 1987 la part des moyens de production dans la production globale était peu différente de celle de l'URSS ou des USA ${ }^{6}$ : seuls des pays disposant d'un marché de plusieurs centaines de millions de consommateurs peuvent se permettre une structure économique comparable à celle de la petite Bulgarie socialiste. Les effets de cette myopie économique auront des effets dramatiques de longue durée. 
... et une situation écologique préoccupante régime ont été en Bulgarie le fait d'un mouvement écologique spontané, provoqué par les pollutions chlorées relevées à Rusé et provenant de l'usine chimique roumaine de Giurgiu, de l'autre côté du Danube. Et de graves problèmes écologiques existent dans les grandes villes et les centres industriels et miniers. Qu'est-ce qui a provoqué la détérioration de l'environnement en Bulgarie? D'abord c'est le modèle de développement basé sur l'industrie lourde, grosse consommatrice d'énergie et de matières premières, modèle d'autant plus contestable qu'il s'agissait d'un petit pays sans tradition industrielle lourde, pauvre en énergie et matières premières, et qu'il impliquait l'utilisation de gisement minéraux de faible teneur. équipements soviétiques - est une autre cause d'aggravation de la pollution. La plupart des usines ont été construites sans installation d'épuration. Aujourd'hui environ $70 \%$ de la pollution de l'air en Bulgarie provient du pays même, les $30 \%$ restants venant des pays situés à l'ouest (Italie, Serbie et Montenegro), mais aussi de Roumanie et d'Ukraine. Presque tous les cours d'eau, dans leur cours moyen et inférieur, sont atteints également, et la Bulgarie exporte ses eaux polluées vers la Grèce. Un tiers de la population (incluant toutes les agglomérations urbaines) vit dans des conditions de pollution de l'air constamment au-dessus des plafonds admis. Il est cependant difficile de comparer avec les pays occidentaux parce que, pour la plupart des polluants, les normes bulgare sont plus sévères. de Kozlodui : sur ses six tranches, quatre sont dans de mauvaises conditions techniques et le personnel qualifié n'est pas suffisant. Outre l'amélioration de la sécurité et la modernisation du site existant, la question d'une deuxième centrale à Svichtov est aujourd'hui posée.

Assurer le passage à l'économie de marché : avec qui et pour qui ?

donc la situation est très difficile, et tout pronostic hasardeux, car tout est réversible, sauf le passage à l'économie de marché. Mais qui le fera et qui profitera de ce passage, c'est la question fondamentale. Car le Parti socialiste (ex-communiste) a su se transformer en une couche de nouveaux capitalistes avec des ressources provenant de l'État ou des entreprises pendant les dernières années du régime précédent. Le problème est compliqué par le fait que les cadres compétents en matière de gestion relevaient aussi de la nomenklatura, et que les soucis de promotion personnelle et les luttes pour le pouvoir ont quelque peu brouillé les cartes.

La priorité économique du pays est le passage à l'économie de marché, et la stratégie adoptée a été celle de la thérapie de choc. Pour le gouvernement et les spécialistes étrangers, les secteurs clefs pour la réforme économique sont l'agriculture, l'agroalimentaire et le tourisme. Après les événements de 1989 et même dans certains cas avant, un processus de privatisation a commencé, qui touche la plupart des banques, des services, du commerce de détail, de la restauration et de l'artisanat.

restitution des terres agricoles reste un processus difficile qui, après l'adoption des lois foncières de 1991 et 1992, devrait aboutir et permettre la formation d'un marché des terres agricoles. Mais la crise de l'agriculture est grave, notamment dans le secteur de l'élevage, où le déficit en fourrages a entraîné l'abattage de dizaines de milliers de têtes. La création de nouvelles coopératives agricoles de propriétaires et de 
coopératives de commercialisation sont attendues comme une nécessité par un grand nombre de paysans.

Une grande partie de l'industrie et notamment des industries lourdes n'est pas encore privatisée, mais la location ou la gérance des entreprises s'est largement répandue. A la fin 1991, 150000 firmes privées avaient été enregistrées dans le pays, mais 10 \% d'entre elles seulement avaient obtenu des résultats financiers positifs.

Les effets de cette thérapie de choc, qui pèse lourdement sur la population, ont amené récemment le Parlement à prendre quelques distances vis-à-vis du gouvernement de Filip Dimitrov, qui a dû démissionner. Dans le même temps le Président s'est prononcé à plusieurs reprises pour la «concorde nationale ", ce qui supposerait que quelques inflexions soient apportées à la politique appliquée depuis deux ans.

Le problème démographique, question cruciale

21 Mais ces graves problèmes économiques ne doivent pas cacher ce qui m'apparaît comme une question cruciale en cette fin de siècle: en 1990 et 1991, le taux d'accroissement naturel de la population est pour la première fois (périodes de guerre exceptées) négatif. On estime habituellement que près de 300000 citoyens bulgares ont quitté le pays (essentiellement des Turcs et de jeunes Bulgares) durant les années 89, 90 et 91.

22 Environ $70 \%$ de la population bulgare est urbaine. Pendant les années cinquante, soixante, soixante-dix et quatre-vingt, un fort exode rural a profondément perturbé le pays. Un processus de vieillissement touche la campagne bulgare, où plus de $30 \%$ de la population (contre $20 \%$ environ dans l'ensemble du pays) atteint l'âge de la retraite, à l'exception des régions de population musulmane. Dans le nord, l'ouest et la Strandja, il existe des régions rurales pratiquement dépeuplées. Le comportement démographique de la population turque (environ 800000 personnes, essentiellement localisées dans les Rhodopes orientales et la Bulgarie du nord-est) est totalement différent : ils quittent rarement leurs villages et leur taux d'accroissement naturel est deux à trois fois plus élevé que chez les Bulgares. La politique de bulgarisation des noms a été une des graves erreurs de T. Jivkov : il en est résulté une émigration de plus de 300000 personnes en Turquie, dont une moitié seulement est revenue après la chute du régime. Durant l'année 1992 l'exode a repris, sur une base économique cette fois, et selon le dernier recensement, (décembre 1992) la population bulgare serait tombée à 8.470 .000 habitants, soit un demi-million de moins qu'en 1988.

Caractérisés également par un taux élevé d'accroissement naturel, les Tziganes constituent une minorité nombreuse (que l'on estime à un demi-million environ), mais il s'agit surtout d'une population urbaine, localisée à la périphérie des villes. L'existence des "pomaks ", population bulgarophone islamisée de force au XVII e siècle (environ 300000 habitants regroupés dans les Rhodopes occidentales) est une spécificité bulgare : les dernières élections ont révélé qu'ils votaient massivement pour le MDL, expression de la minorité dite « de conscience turque $»^{7}$.

Une nouvelle géographie électorale

Après une longue et rigide dictature de type soviétique, les changements révolutionnaires de 1989 ont débouché sur une vie politique très intense et pluraliste. En général, on observe une claire bipolarisation de la société et une grande politisation des masses autour des causes de la crise, de la culpabilité de l'élite communiste et de la 
nomenklatura, et surtout sur la question des voies possibles pour un nouveau développement.

Environ 120 partis ont été enregistrés, mais les plus importants sont au nombre de 7 ou 8 , dont seulement trois (UFD, PSB et MDL) sont représentés à l'Assemblée nationale.

Figure 2 : LES FORCES POLITIQUES EN BULGARIE AUX SCRUTINS DE 1990 ET $1991^{8}$

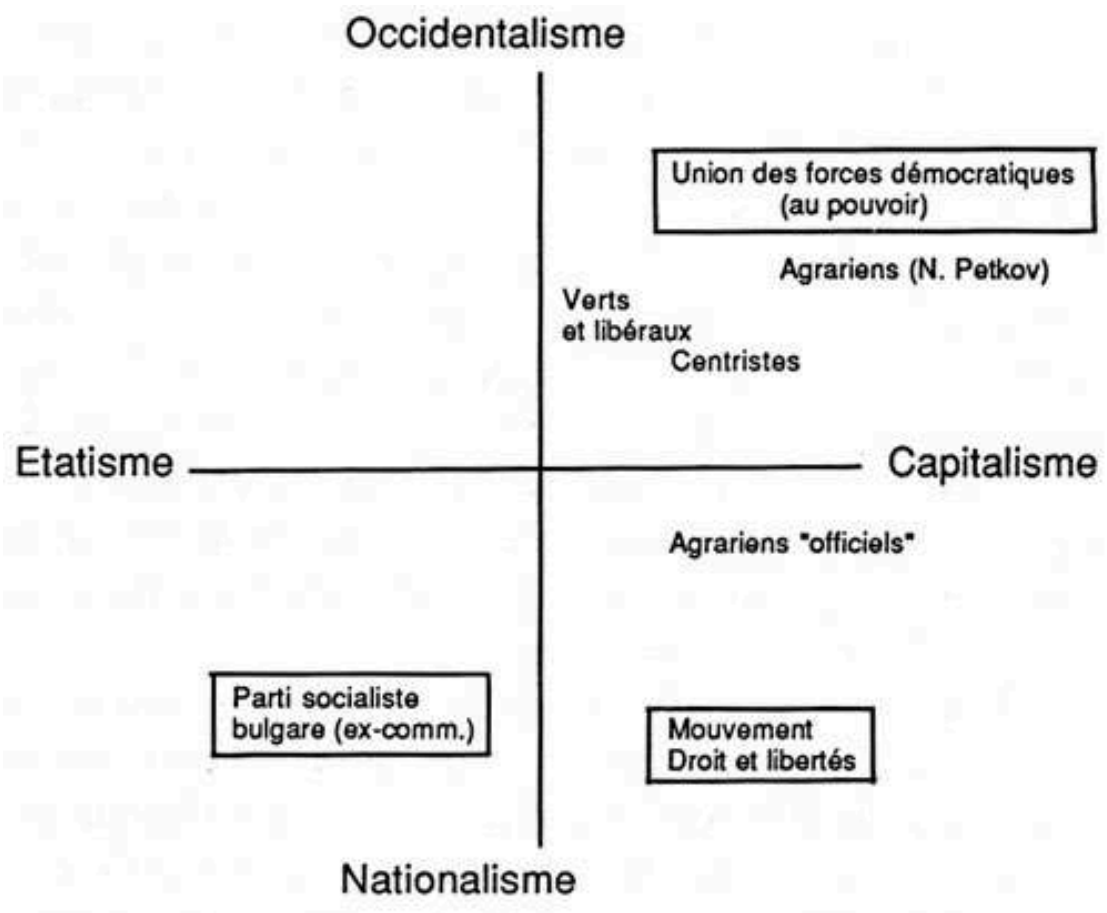

(Les partis représentés au Parlement depuis octobre 1991 sont encadrés. )

L'Union des Forces démocratiques a gagné les dernières élections législatives avec une petite marge de 1,5\% de voix de plus que le PSB. L'UFD regroupe des partis différents, certains plutôt centristes, mais aussi un groupe de partis clairement orientés à droite. Les bastions de l'UFD sont les grandes villes, les régions montagneuses de tradition industrielle (comme Gabrovo par exemple) et la Macédoine du Pirin. Ce dernier point mérite une explication. Durant les années 1945-47, selon un accord survenu entre Staline et Tito, cette partie bulgare de la Macédoine ( $15 \%$ du territoire et $10 \%$ de la population de la Macédoine historique) dut subir une politique de macédonisation, vécue par la population, qui s'est toujours sentie bulgare, comme une trahison du parti communiste bulgare de l'époque, et qu'elle n'a pas oubliée?

Le deuxième parti, le Parti Socialiste Bulgare (ex-communiste) est lui aussi divisé entre différents courants, "progressistes » d'une part, et « durs », soutenus par la masse des adhérents, d'autre part. Ce parti jouit d'une grande popularité dans les régions agricoles des plaines danubiennes et de la Thrace. La plupart des retraités vote aussi pour le PSB, mais curieusement pas la classe ouvrière. Les mêmes observations peuvent être faites pour les élections présidentielles, où le candidat soutenu par le PSB, resté seul en lice contre J. Jelev au deuxième tour, a rassemblé 48,5\% des voix (figure 3).

Le Mouvement pour les Droits et les Libertés est une formation politique qui regroupe les musulmans bulgares. Du fait de l'élimination de nombreux petits partis, le MDL a pu lors des dernières élections remporter $11 \%$ des sièges à l'Assemblée nationale avec 
$7,5 \%$ des suffrages. L'UFD n'ayant pas la majorité absolue, le MDL lui a apporté son soutien, décisif pour faire accepter les lois foncières et celles concernant la privatisation, qui sont cruciales pour le passage à l'économie de marché. Cette position clef du MDL est rejetée par les nationalistes bulgares. Dernière force politique traditionnelle importante, les partis agrariens ont été victimes de leurs divisions, aucun des deux principaux (Parti Agrarien Bulgare unifié et PAB-Nikola Petkov) n'ayant atteint la barre des $4 \%$ nécessaires pour être représentés au Parlement.

Dernièrement, du fait des pénuries, une déception s'est fait jour dans l'opinion à l'égard du gouvernement au pouvoir, et les tendances monarchistes s'en sont trouvées renforcées. Presque tous les partis ont d'ailleurs établi des contacts avec l'homme d'affaires prospère, résidant en Espagne, qu'est l'héritier de la couronne et candidat au trône Siméon II. Mais il n'y a pas de députés élus sous l'étiquette monarchiste.

La comparaison entre les élections de 1991 et 1992 montre un lent changement de l'opinion publique en faveur de partis de droite et centristes. Mais plus de $30 \%$ de l'électorat reste contrôlé par le PSB. Le problème est que ce parti n'a pas un programme établi (il reprend les postulats de l'UFD). La nomenklatura qui était un prolongement économique du PC est en train de devenir, dans le nouveau contexte politique et économique, une couche capitaliste puissante. Et c'est une question de morale qui divise la société : le passage à l'économie de marché doit-il être fait par les privilégiés de l'ancien régime?

Conclusion

31 La Bulgarie est du point de vue géographique, historique et socioculturel un pays de transition entre l'Europe et le Proche-Orient. Mais c'est aussi un pays qui, à une période historique difficile et au travers d'une crise économique, politique et morale extrêmement grave, opère sa transition du modèle administratif et centralisé vers une société ouverte et libérale. Cette situation transitoire, qui affecte le pays dans toutes ses dimensions, contribue à la complexité et à la fragilité des processus en cours.

Les bouleversements profonds que connaît la Bulgarie comme les autres pays de l'Est sont équivalents à une vraie révolution sociale. Heureusement les principales forces politiques et surtout le peuple de notre pays ont su éviter les conflits ouverts qui ont touché les autres pays balkaniques ex-socialistes. C'est là l'apport le plus précieux et l'originalité de la voie bulgare pour l'Europe de l'avenir.

Conf. Figure 3 en document annexe : Elections présidentielles de janvier 1992

Figure 4 : BULGARIE : QUELQUES DONNéES

\begin{tabular}{|l|l|}
\hline & Population d'âge actif : \\
\hline 1948 & 4243000 \\
\hline \hline 1980 & 5087000 \\
\hline 1987 & 5005000 \\
\hline 1992 & 4800000 \\
\hline
\end{tabular}


- Part des moyens de production dans la production globale en \% (1987) :

URSS : 75 / USA : 68 / Bulgarie : 62

- Produit matériel brut et emploi par secteurs économiques en \% (1987) :

\begin{tabular}{|l||l|l|}
\hline Secteur & Produit matériel brut & Emplois \\
\hline \hline Agriculture & 12,6 & 20,7 \\
\hline \hline Industrie & & 35,0 \\
\hline \hline Construction & 9,6 & 8,6 \\
\hline \hline Transport & 7,6 & 7,4 \\
\hline \hline Commerce & 7,5 & 9,0 \\
\hline \hline Autres & 2,1 & 19,1 \\
\hline \hline Total & 100,0 & 100,0 \\
\hline
\end{tabular}

\section{NOTES}

1. La doctrine du containment, ou endiguement, formulée à l'origine en juillet 1947 par le diplomate Georges Kennan dans la revue Foreign Affairs, puis reprise par Marshall et Foster Dulles, fut à la base de la politique internationale des Etats-Unis : assuré par le maintien de troupes militaires en Europe et en Extrême-Orient, le containment était destiné à opposer à l'URSS et à ses alliés « une force inaltérable en tout point où ils montreront des signes de leur volonté d'empiéter sur les intérêts d'un monde pacifique et stable. » $(\mathrm{NdE})$

2. Le géographe britannique H. J. MACKINDER (1861-1947) définissait un heartland continental centré sur la Russie, héritière d'une tradition gréco-slave, un croissant semi-continental, et un croissant externe insulaire. L'article fondateur de sa théorie The Geographical Pivot of History (1904) - a été réédité par E. W. Gilbert en 1951 (Londres, BRSG).

3. Cf. G. PREVELAKIS, 1990, «La maison européenne commune : une maison hantée », Acta Geographica, $\mathrm{n}^{\circ}$ 1, 1990.

4. Cf. la récente réédition du célèbre reportage d'Albert LONDRES, « Les Comitadjis », 1932, in Oeuvres complètes, Arléa, Paris, 1992, pp. 785-850. 
5. Une nation macédonienne s'est bien constituée dans la République yougoslave de Macédoine, mais cela n'a rien à voir avec la population restée en Bulgarie (Cf. G.

NAIDENOV, 1991, Kakvo stava? (Qu'est-ce qui se passe ?), Sofia.

6. Cf. tableau infra.

7. La constitution interdit en effet l'existence de formations politiques à base ethnique ou religieuse.

8. Schéma repris et adapté de J. VANLAER, 1990, « Les premières élections libres en Europe de l'Est : systèmes de partis et clivages régionaux ", Revue belge de géographie, 1991, pp. 142-158.

9. Cf. Marin BATCHVAROV, 1993, The electoral geography of the post communist Bulgaria, Communication au séminaire international de géopolitique, Sofia, octobre 1992.

\section{RÉSUMÉS}

Dans un espace balkanique à nouveau confronté à des conflits d'une grande violence, la Bulgarie opère de manière pacifique sa transition d'un modèle administratif et centralisé à une société ouverte et libérale, tout en préservant avec ses voisins des relations de bon voisinage. Cette mutation s'opère toutefois dans les conditions d'une crise profonde: déformations héritées de l'ancien mode de développement, problème démographique, situation écologique préoccupante. Depuis 1991, la Bulgarie s'est attachée à suivre les prescriptions des institutions financières internationales, et son association à la CEE, ardemment souhaitée par la plupart des formations politiques, est désormais acquise. Mais la chute de la production - aggravée par la rupture des débouchés traditionnels - et la politique de privatisation ont entraîné une forte poussée du chômage et creusé les inégalités sociales : la question centrale est donc de savoir par qui et au profit de quelles couches le changement social s'opère. Après deux années de "thérapie de choc ", la géographie électorale bulgare reste caractérisée par la polarisation autour de deux grandes forces politiques (Union des Forces Démocratiques et Parti Socialiste Bulgare).

Bulgaria today: A Balkan state unlike the others?

Since 1989, the Balkan states have once again been dominated by violence, riots and wars. Among them, Bulgaria seems to be the exception. The transition to capitalism is proceeding in a conflictual but peaceful way. Beyond its frontiers, normal relations are being developed with neighbouring countries. The great hope of becoming a member of the European Economic Community has been partly fulfilled by the association agreement of december 22th (1992). But the crisis is profound, the main issues being the socialist inheritance, the demographic problems, the ecological situation. The new leaders have paid great attention to following the IMF and World Bank prescriptions. But commercial links with former CMEA nations have been disrupted, production has fallen and privatization has increased social inequalities. The question is by whom and for whom will social change be conducted. After two years of "shock therapy ", the electoral landscape remains dominated by two main political forces, the Union of Democratic Forces and the Bulgarian Socialist Party. 
INDEX

Mots-clés : Environnement, Privatisation, modèle de développement, Géographie électorale, Géopolitique, Intégration européenne, Transition, Bulgarie, Balkans

Keywords : Privatization, development model, Ecology, Electoral geography, European integration, Bulgaria, Balkan states

\section{AUTEUR}

\section{MARIN BATCHVAROV}

Est professeur de géographie économique à l'Université Saint-Clément d'Ohrid de Sofia, où il dirige depuis 1980 la chaire de géographie du tourisme à la Faculté de géographiegéologie. Il vient d'être nommé Vice-doyen de la Faculté d'économie. 